Bundesgesundheitsbl -

Gesundheitsforsch - Gesundheitsschutz

2000 • 43:163-164 @ Springer-Verlag 2000

\section{Editorial}

B.-M. Bellach

Robert Koch-Institut, Berlin

\title{
"Guten Tag, wie geht es Ihnen?"
}

\section{Liebe Leserinnen, liebe Leser,}

die Antwort auf die obige Frage wird davon abhängen, wer sie fragt und wie ernst die Frage gemeint sein könnte, ob Sie gerade Ärger hatten, und wie gut Sie letzte Nacht geschlafen haben. Auf dieselbe Frage Ihres Hausarztes werden Sie sicherlich anders antworten als auf die freundliche Nachfrage eines Kollegen oder die Ihres Chefs. Es gibt auch kulturelle Unterschiede im Herangehen an diese Frage. Es sei hier nur an das formale „How are you?” der Amerikaner gedacht, letztere wären höchst erstaunt über einen ernsthaften Versuch, diese Frage präzise zu beantworten.

Aber nun hinterfragen Sie doch einmal innerlich sich selbst, wie es Ihnen geht. Es werden verschiedene Komponenten Ihres Befindens überprüft, wie etwa das körperliche oder seelische Befinden, aber auch das sich wohl fühlen in Familie, Beruf und Bekanntenkreis, und Sie werden Ihre Lebensumstände ins Verhältnis setzen zu anderen Personen mit vergleichbaren Rahmenbedingungen.

Das alles läuft in Sekundenschnelle vor Ihrem geistigen Auge ab, und Sie werden gegebenenfalls antworten: „Danke, mir geht es gut” oder „Danke, mir geht es schon besser” oder „Danke, es geht mir derzeit nicht so gut". Lässt sich nun aber Ihre Antwort mit der Ihres Nachbarn vergleichen und ist die Tatsache, dass es Ihnen nach eigenen Angaben schlecht geht, eigentlich da-

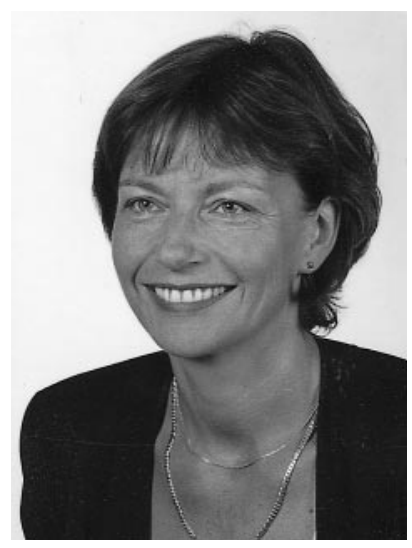

durch bedingt, dass Sie krank sind oder dass Sie Schmerzen oder Kummer oder schlechte Laune haben?

Um dies herauszufinden, haben Psychologen, Soziologen, Mediziner und diverse andere Berufsgruppen verschiedene Messinstrumente entwickelt, die auf recht trickreiche Art versuchen, aus dem Befragten, ohne Misstrauen bei ihm aufkommen zu lassen, herauszubekommen, wie es ihm denn so geht. Das ganze heißt dann Messung der gesundheitsbezogenen Lebensqualität und bekommt die schicke Abkürzung HRQOL für die englische Version „Health Related Quality of Life".

$\mathrm{Zu}$ HRQOL gibt es inzwischen umfangreiche Literatur. Ein Review-Artikel in diesem Heft soll einen Überblick darüber geben und den Einstieg in vertiefende Studien erleichtern. Regelmäßig durchgeführte große Konferenzen (über eine wird in diesem Heft berichtet) und verschiedene Anwendungen der inzwischen getesteten und in verschiedene Sprachen übersetzten Instrumente legen Zeugnis ab vom Fortschritt auf dem Gebiet der Lebensqualitätsforschung. Das ursprüngliche Anwendungsgebiet der „HRQOL-Messinstrumente" war die Therapie-bzw. Evaluationsforschung. Die Lebensqualität vor und nach einer Therapie ist ein überprüfbarer Indikator des Behandlungserfolges. Die Brauchbarkeit der gängigen Messinstrumente zur Erfassung von Veränderungen in der gesundheitsbezogenen Lebensqualität ist vielfach unter Beweis gestellt. Hier sind die entsprechenden Fragebatterien bereits in der Routineanwendung. Zwei aktuelle Beispiele hierfür, Lebensqualität unter Dialyse und nach Nierentransplantation sowie Lebensqualität nach Herzoperationen, fanden leider nicht mehr in diesem, aber dafür im nächsten Heft von „Bundesgesundheitsblatt Gesundheitsforschung Gesundheitsschutz" Platz.

Eines der am häufigsten angewendeten Messinstrumente, der SF-36 (Short Form 36) fand nunmehr auch Anwendung auf Bevölkerungsebene. 7124 Teilnehmer des Bundes-Gesundheitssurveys 1998 beantworteten die 36 Fragen des SF-36. Dabei wurde die Antwort auf die Frage „Wie geht es den erwachsenen Deutschen?"gesucht. Wie

Dr. Bärbel-Maria Bellach Robert Koch-Institut, Postfach 650280, 13302 Berlin 
die Antwort aussieht, und welche zusätzlichen Erkenntnisse bei der Auswertung des Zusammenhanges zwischen den SF 36-Werten und den parallel da$z u$ erfassten Gesundheitsparametern gewonnen wurden, finden Sie ebenfalls in diesem Heft.

Kinder nach ihrem Befinden zu befragen, erfordert ganz besondere Überlegungen. Zum einen müssen Fragen kürzer und anders gestellt werden, zum anderen ist gesundheitsbezogene Lebensqualität im Kindesalter anders $z u$ definieren als bei Erwachsenen. Welche Methoden für dieses Alter bislang ausgetestet wurden und mit welchen Ergebnissen, ist auch in diesem Heft nachzulesen.
Die WHO definiert Gesundheit als Zustand vollständigen Wohlbefindens in physischer, psychischer und sozialer Hinsicht. Die in diesem Heft beleuchteten Zugänge zur Erfassung gesundheitsbezogener Lebensqualität tragen dieser Definition Rechnung. Es geht nicht darum, das Vorhandensein oder Fehlen von Krankheit oder die Lebenserwartung zum Zielkriterium zu machen, sondern es geht um das individuelle Wohlbefinden. Wie sehr dieses durch aktuelle Nachteile wie akuten Schmerz beeinträchtigt werden kann, zeigen die Auswertungen des Bundes-Gesundheitssurveys. Es ergibt sich aus diesen Daten aber genauso gut, dass Unzufriedenheit allgemein sehr stark mit der gesundheitlichen Befindlichkeit zusammenhängt.

Der SF-36 als ein Messinstrument zur Erfassung gesundheitsbezogener Lebensqualität ist damit ein Seismograph, der auf verschiedenste Einflüsse und Faktoren im Leben eines Menschen reagiert. Offen bleibt die Frage, ob dieses Instrument nun die Wünschelrute ist, mit der auf recht einfache Weise alles zu erfassen ist, was im Zusammen- hang mit Gesundheit steht, mit der vielleicht sogar Prognosen über Inanspruchnahme medizinischer Leistungen oder Arbeitsunfähigkeit möglich werden. Die Weiterentwicklung der Instrumente zur Erfassung gesundheitsbezogener Lebensqualität auf Bevölkerungsebene, nicht nur bei Patientengruppen, bleibt ein Erfordernis.

Sie sehen also, wie viel Aufwand betrieben wird, um herauszufinden, $o b$ es Ihnen wirklich gut geht. Antworten Sie bitte deshalb sorgsam auf die eingangs gestellte Frage, schließlich stehen ganze Theorien hinter Ihrer Antwort, selbst wenn Sie das möglicherweise bislang noch gar nicht gemerkt haben.

Ihre
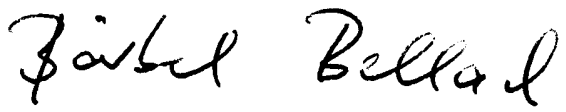

B.-M. Bellach 